\title{
Atomic Resolution Electron Tomography on a Discrete Grid: Atom Count Errors
}

\author{
K.J. Batenburg, ${ }^{*}$ J.R. Jinschek, ${ }^{* *}$ and C. Kisielowski** \\ * Leiden University, Mathematical Institute, P.O. Box 9512, 2300 RA, Leiden, The Netherlands and \\ Centrum voor Wiskunde en Informatica, P.O. Box 94079, 1090 GB, Amsterdam, The Netherlands. \\ ** Ernest Orlando Lawrence Berkeley National Laboratory, National Center for Electron \\ Microscopy, 1 Cyclotron Road MS 72R0150, Berkeley, CA 94720, U.S.A.
}

The resolution of state-of-the-art electron tomography is in the nm range. In a new approach [1], atomic resolution was achieved by determining the positions of all atoms in a nanocrystal (Fig. 1) from a small number of electron tomographic projections assuming that the atoms lie on a discrete grid. The exit wave is reconstructed for each of the projections and the number of atoms in each projected column is subsequently determined using channeling theory [2]. The discrete projection data, consisting of the number of atoms in each projected column of the discrete grid, is used as input for a discrete tomography reconstruction algorithm [3, 4], which computes the desired 3D reconstruction. It has been demonstrated [1] that the 3D shape of a 309-atom nanocrystal (Fig. 1) could be reconstructed exactly from only three projections. In this case, the process of counting the number of atoms was assumed to be free of errors. However, count errors are practically realistic and occur when the number of atoms in a column is counted incorrectly, e.g. due to noise.

The algorithm for computing reconstructions on a discrete grid $[3,4]$ is capable of dealing with these count errors in the projection data, but for such input data the resulting reconstruction will usually deviate slightly from the measured object. The main interest for the current application of nanocrystal reconstruction is to obtain perfect reconstructions, even in the presence of count errors. Therefore, we included a postprocessing step. It better approximates the projection data by repeatedly locating (and modifying) grid cells for which adding (or deleting) an atom improves the current approximation. When no such grid cells can be found, the procedure terminates and outputs the reconstruction.

To test our new algorithm, we started with perfect projections (without count errors) of the nanocrystal from [1]. A number of count errors was added to the projection data by repeatedly selecting a random projected column and adding +1 or -1 (both with $50 \%$ probability) to the number of atoms in that particular column (compare Fig. 1c and 1d).

By adding only a few extra projections, the tomographic reconstruction procedure can be made far more resilient to count errors. If more projections are available, the set of measured projections contains a certain amount of redundancy, since a subset of the available projections is already sufficient to compute all atom positions (in the case of error-free projections). It is often possible to exploit this redundancy in order to compute perfect reconstructions even in the presence of count errors. Moreover, if a sufficient number of projections is available, a large number of errors can be corrected. The ability to correct a large number of count errors enforces our expectation that the method can also be applied to real-world data, instead of simulated data. The idea of using redundancy for correcting errors originates from the mathematical field of coding theory, where one tries to find ways of encoding a digital signal so that it can be sent along a noisy transmission channel and still be decoded without errors by the receiver.

Table 1 summarizes the reconstruction results for varying numbers of available projections and varying numbers of count errors. For every case, 100 test runs were performed, each time with 
different, randomly generated count errors. The number of atom errors for each reconstruction was determined by counting the number of grid cells in the reconstruction that are different from the original crystal. The results demonstrate that the algorithm is well capable of correcting count errors. Increasing the number of projections significantly increases the tolerable number of errors. When 80 count errors are made, the algorithm still reconstructs the crystal perfectly in 39\% of the cases if 6 projections are available.

The principle of the algorithm, its performance, requirements and limitations will be presented. How the approach can be used when working with several different types of atoms will also be discussed.

\section{References}

[1] J.R. Jinschek et al., Microsc. Microanal. 10 (Suppl 3) (2004) 44-45.

[2] J.R. Jinschek et al., Proceedings of SPIE 5187 (2004) 54.

[3] K.J. Batenburg, CWI Research Report PNA-E0418 (2004, submitted).

[4] K.J. Batenburg, Proceedings of the Workshop on Discrete Tomography and its Applications, New York (2005, to appear).

[5] This work was supported by the Director, Office of Science, of the US Department of Energy under the contract DE-AC03-76SF00098. One author (J.J.) acknowledges support of the Alexander von Humboldt-Foundation, Bonn/Germany. One author (K.J.B.) acknowledges support of the Netherlands Organization for Scientific Research (NWO) under contract 613.000 .112 .

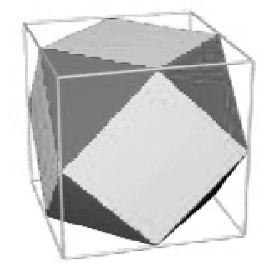

a)

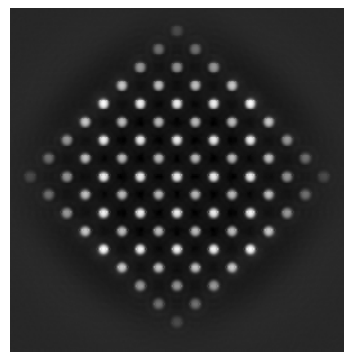

b)

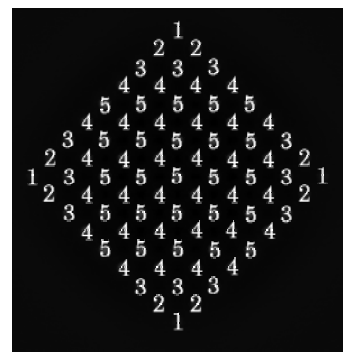

c)

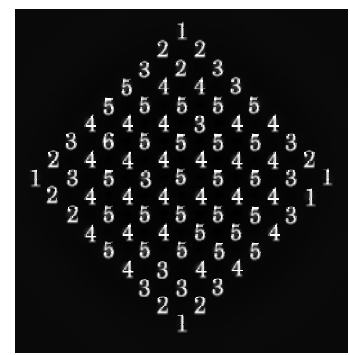

d)

Figure 1. a) 309 atom cuboctahedron shaped nanocrystal (schematic drawing)

b) EW phase image in a $<001>$ zone axis

c) Number of atoms in each column without count errors

d) Number of atoms in each column with several count errors

Table 1. Reconstruction results for varying numbers of available projections and count errors.

\begin{tabular}{|c|c|c|c|c|c|c|c|c|c|c|c|c|}
\hline \#projections & \multicolumn{4}{|c|}{3} & \multicolumn{4}{|c|}{5} & \multicolumn{4}{|c|}{6} \\
\hline orientations & \multicolumn{4}{|c|}{ [001], [110], [110] } & \multicolumn{4}{|c|}{$\begin{array}{c}{[001],[110],[110],} \\
{[011],[111]}\end{array}$} & \multicolumn{4}{|c|}{$\begin{array}{l}{[001],[110],[110],} \\
{[011],[111],[1 \underline{1} 1]}\end{array}$} \\
\hline \#count errors & 10 & 20 & 40 & 80 & 10 & 20 & 40 & 80 & 10 & 20 & 40 & 80 \\
\hline$\%$ perfect & 36 & 3 & 0 & 0 & 100 & 97 & 61 & 6 & 100 & 99 & 80 & 39 \\
\hline avg. \#atom errors & 1.28 & 4.51 & 11.92 & 27.13 & 0 & 0.03 & 0.57 & 3.73 & 0 & 0.01 & 0.23 & 1.18 \\
\hline
\end{tabular}

